\title{
HIGH-DISCREPANCY SEQUENCES
}

\author{
Shu TEZUKA
}

(Received 30 June 2006)

\begin{abstract}
First, it is pointed out that the uniform distribution of points in $[0,1]^{d}$ is not always a necessary condition for every function in a proper subset of the class of all Riemann integrable functions to have the arithmetic mean of function values at the points converging to its integral over $[0,1]^{d}$ as the number of points goes to infinity. We introduce a formal definition of the $d$-dimensional high-discrepancy sequences, which are not uniformly distributed in $[0,1]^{d}$, and present motivation for the application of these sequences to high-dimensional numerical integration. Then, we prove that there exist non-uniform $(\infty, d)$-sequences which provide the convergence rate $O\left(N^{-1}\right)$ for the integration of a certain class of $d$-dimensional Walsh function series, where $N$ is the number of points.
\end{abstract}

\section{Introduction}

It is well known [3] that the uniform distribution of points $X_{n}, n=0,1, \ldots$, in $[0,1]^{d}$ is a necessary and sufficient condition for every function $f\left(x_{1}, \ldots, x_{d}\right)$ in the class of all Riemann integrable functions over $[0,1]^{d}$, hereafter denoted by $\Re_{d}$, to satisfy

$$
I(f):=\int_{[0,1]^{d}} f\left(x_{1}, \ldots, x_{d}\right) d x_{1} \ldots d x_{d}=\lim _{N \rightarrow \infty} \frac{1}{N} \sum_{n=0}^{N-1} f\left(X_{n}\right) .
$$

It is also known [3] that for the class of all continuous functions over $[0,1]^{d}$, which is a proper subset of $\Re_{d}$, the uniform distribution is a necessary and sufficient condition for every function in the class to satisfy equation (1). The well-known

2000 Mathematics Subject Classification: Primary 65D30.

Keywords and Phrases: discrepancy; high-dimensional numerical integration; $(t, d)$-sequences; Walsh functions.

This article is an invited contribution to a special issue of the Kyushu Journal of Mathematics commemorating the sixtieth volume. 
Koksma-Hlawka theorem implies that the class of all functions over $[0,1]^{d}$ of bounded variation in the sense of Hardy and Krause is another proper subset of $\Re_{d}$ for which the uniform distribution is also a necessary and sufficient condition for every function in the class to satisfy equation (1).

However, little is known about what happens to any other proper subsets of $\mathfrak{R}_{d}$. Although the uniform distribution of sample points is a sufficient condition for every function in any proper subset of $\Re_{d}$ to satisfy equation (1), it is not always a necessary condition. The purpose of this paper is to explore a possibility to develop faster algorithms for a proper subset of $\Re_{d}$ based on non-uniform sample points than Monte Carlo and/or quasi-Monte Carlo methods. In the next section, we first introduce a formal definition of the $d$-dimensional high-discrepancy sequences, which are not uniformly distributed in $[0,1]^{d}$, and present motivation for the application of these sequences to high-dimensional numerical integration. Then, we prove that there exist non-uniform $(\infty, d)$-sequences which provide the convergence rate $O\left(N^{-1}\right)$ for the integration of a certain class of $d$-dimensional Walsh function series, where $N$ is the number of points. In the last section, we discuss the significance of this result and future research directions.

\section{Main result}

\subsection{Discrepancy and $(t, d)$-sequences}

For a point set $P_{N}=\left\{X_{0}, X_{1}, \ldots, X_{N-1}\right\}$ of $N$ points in $I^{d}=[0,1]^{d}$ and a subinterval $J$ of $I^{d}$, we define $A(J, N)$ as the number of $n, 0 \leq n \leq N-1$, with $X_{n} \in J$ and $V(J)$ is the volume of $J$. Then the discrepancy of $P_{N}$ is defined by

$$
D_{N}^{(d)}=\sup _{J}\left|\frac{A(J, N)}{N}-V(J)\right|,
$$

where the supremum is taken over all subintervals $J$ of the form $\prod_{i=1}^{d}\left[0, y_{i}\right)$. For more mathematical detail on discrepancy, see, e.g., $[\mathbf{1}, \mathbf{4 - 7}]$.

An elementary interval in base $b(b \geq 2)$ is a subinterval of $I^{d}$ of the form

$$
E=\prod_{i=1}^{d}\left[a_{i} b^{-k_{i}},\left(a_{i}+1\right) b^{-k_{i}}\right) \quad \text { with } 0 \leq a_{i}<b^{k_{i}} \text { and } k_{i} \geq 0 .
$$

A $(t, m, d)$-net in base $b$ is a point set of $b^{m}$ points in $I^{d}$ such that $A\left(E, b^{m}\right)=b^{t}$ for every elementary interval $E$ in base $b$, with $V(E)=b^{t-m}(0 \leq t \leq m)$. A $(t, d)$ sequence in base $b$ is an infinite sequence, $X=\left(X_{n}\right)_{n \geq 0}$, of points in $I^{d}$ such that for 
all $h \geq 0$ and $m>t$, the point set $\left\{\left[X_{h b^{m}}\right]_{m}, \ldots,\left[X_{(h+1) b^{m}-1}\right]_{m}\right\}$ is a $(t, m, d)$-net, where $\left[X_{n}\right]_{m}$ denotes the coordinate-wise $m$-digit truncation of a point $X_{n}$.

The general construction principle of $(t, d)$-sequences in base $b$ is as follows:

(i) $R$ is a commutative ring with $\operatorname{card}(R)=b$ and $B=\{0,1, \ldots, b-1\}$;

(ii) for an integer $r \geq 1$, choose bijections $\psi_{r}$ from $B$ to $R$ with $\psi_{r}(0)=0$ for all sufficiently large $r$;

(iii) for integers $i, j$ such that $1 \leq i \leq d$ and $j \geq 1$, choose bijections $\lambda_{i, j}$ from $R$ to $B$;

(iv) for integers $i, j, r$ as above, choose elements $c_{j, r}^{(i)} \in R$.

If $n=\sum_{r=1}^{\infty} a_{r}(n) b^{r-1}$ is the $b$-ary expansion of $n \geq 0$, set $X_{n}^{(i)}=\sum_{j=1}^{\infty} x_{n, j}^{(i)} b^{-j}$, where $x_{n, j}^{(i)}=\lambda_{i, j}\left(\sum_{r=1}^{\infty} c_{j, r}^{(i)} \psi_{r}\left(a_{r}(n)\right)\right)$ for $1 \leq i \leq d$. Here, the matrices $C^{(i)}=\left(c_{j, r}^{(i)}\right)_{j \geq 1, r \geq 1}$ are called the generator matrices of $X$. Then, the sequence $X=\left(X_{n}\right)_{n \geq 0}$, with coordinates $X_{n}^{(i)}$ as above, becomes a $(t, d)$-sequence in base $b$, where the value of $t$ is determined by the linear independence structure of generator matrices. Hereafter, we assume that $b$ is a prime power, and that $R$ in the construction principle is the finite field $G F(b)$. Let

$$
\mathbf{c}_{\ell}^{(i)}(m)=\left(c_{\ell, 1}^{(i)}, \ldots, c_{\ell, m}^{(i)}\right) \in G F(b)^{m},
$$

and let

$$
C\left(k_{1}, \ldots, k_{d} ; m\right)=\left\{\mathbf{c}_{\ell}^{(i)}(m) \mid 1 \leq \ell \leq k_{i}, 1 \leq i \leq d\right\} .
$$

Define $\rho(C ; m)$ as the maximal integer $K$ such that $C\left(k_{1}, \ldots, k_{d} ; m\right)$ is linearly independent over $G F(b)$ for all non-negative integers $k_{1}, \ldots, k_{d}$ satisfying $\sum_{1 \leq i \leq k} k_{i}=K$. We have the following result $[5,7]$.

PROPOSITION 1. If an integer $t \geq 0$ satisfies $t \geq m-\rho(C ; m)$ for each integer $m>t$, then the sequence defined in the above becomes $a(t, d)$-sequence in base $b$.

\subsection{Definition and motivation for high-discrepancy sequences}

First, we introduce the definition of high-discrepancy sequences.

Definition 1. If a sequence of points $X_{n}, n=0,1, \ldots$, in $[0,1]^{d}$ satisfies that for all $N>1$, the discrepancy $D_{N}^{(d)}$ of the first $N$ points is given by

$$
D_{N}^{(d)}=O(1)
$$

then we call it a high-discrepancy sequence, where the implied constant in the $O$ notation depends only on the dimension $d$. 
We should note that if the right-hand side in equation (2) is replaced by $O\left((\log N)^{d} / N\right)$, then it is the definition of a low-discrepancy sequence. Highdiscrepancy sequences are those sequences which are not uniformly distributed, and vice versa. We have the following proposition.

Proposition 2. A $(t, d)$-sequence in base two for which the first rows of its generator matrices are all identical to $(1,0,0, \ldots)$ is a high-discrepancy sequence.

Proof. From Proposition 1, the sequence is an $(\infty, d)$-sequence, i.e. a $(t, d)$-sequence with $t$ being infinity. Since it is distributed in only two sub-domains, $[0,1 / 2]^{d}$ and $[1 / 2,1]^{d}$, the proof follows.

Note that there are two types of $(\infty, d)$-sequences, i.e. uniform or non-uniform. The sequence introduced in Proposition 2 is a non-uniform $(\infty, d)$-sequence.

We give the following theorem, which motivates us to investigate the application of high-discrepancy sequences defined in the above to high-dimensional numerical integration.

THEOREM 1. If an $L_{2}$ function in d dimensions is written as

$$
f\left(x_{1}, \ldots, x_{d}\right)=\sum_{k_{1}, \ldots, k_{d} \geq 0} c_{k_{1}, \ldots, k_{d}} \cos \left(2 \pi k_{1} x_{1}\right) \cdots \cos \left(2 \pi k_{d} x_{d}\right),
$$

then we have

$$
\begin{array}{rl}
\int_{[0,1]^{d}} & f\left(x_{1}, \ldots, x_{d}\right) d x_{1} \ldots d x_{d} \\
= & 2^{d-1}\left(\int_{[0,1 / 2]^{d}} f\left(x_{1}, \ldots, x_{d}\right) d x_{1} \ldots d x_{d}\right. \\
& \left.+\int_{[1 / 2,1]^{d}} f\left(x_{1}, \ldots, x_{d}\right) d x_{1} \ldots d x_{d}\right) .
\end{array}
$$

Proof. Since

$$
\int_{0}^{1} \cos (2 \pi k x) d x=0
$$

for any $k \geq 1$, we have

$$
\int_{[0,1]^{d}} f\left(x_{1}, \ldots, x_{d}\right) d x_{1} \ldots d x_{d}=c_{0, \ldots, 0} .
$$

Since

$$
\int_{0}^{1 / 2} \cos (2 \pi k x) d x=\int_{1 / 2}^{1} \cos (2 \pi k x) d x=0
$$


for any $k \geq 1$, we have

$$
\int_{[0,1 / 2]^{d}} f\left(x_{1}, \ldots, x_{d}\right) d x_{1} \ldots d x_{d}=\frac{c_{0, \ldots, 0}}{2^{d}}
$$

and

$$
\int_{[1 / 2,1]^{d}} f\left(x_{1}, \ldots, x_{d}\right) d x_{1} \ldots d x_{d}=\frac{c_{0, \ldots, 0}}{2^{d}} .
$$

Thus, the proof is complete.

We can also consider the following type of $L_{2}$ functions in $d$ dimensions:

$$
f\left(x_{1}, \ldots, x_{d}\right)=c_{0}+\sum_{k_{1}, \ldots, k_{d} \geq 1} c_{k_{1}, \ldots, k_{d}} \sin \left(2 \pi k_{1} x_{1}\right) \cdots \sin \left(2 \pi k_{d} x_{d}\right) .
$$

It is easy to see that if the dimension $d$ is odd, this class of functions also satisfy equation (3). Recently, Tezuka and Papageorgiou [9] showed that there exists an exact cubature with only one point for this class of functions in any $d$ dimensions.

In what follows, we show that there exist non-uniform $(\infty, d)$-sequences which provide the convergence rate $O\left(N^{-1}\right)$ for the integration of a class of $d$-dimensional Walsh function series.

\subsection{A class of d-dimensional Walsh function series}

The Walsh functions are defined by

$$
\operatorname{wal}(0, x)=1, \quad \text { for } x \in[0,1),
$$

and, for an integer $k \geq 1$,

$$
\operatorname{wal}(k, x)=(-1)^{\sum_{j=1}^{\infty} b_{j-1} a_{j}}=(-1)^{(\mathbf{k}, X)}
$$

where $k=b_{0}+b_{1} 2+\cdots, x=a_{1} 2^{-1}+a_{2} 2^{-2}+\cdots$ in the binary expansion, and $\mathbf{k}=\left(b_{0}, b_{1}, \ldots\right)$ and $X=\left(a_{1}, a_{2}, \ldots\right)$ are the binary vector representation of $k$ and $x$, respectively. Then, we have the following theorem.

THEOREM 2. A function in the d-dimensional $L_{2}$ space can be written as

$$
f\left(x_{1}, \ldots, x_{d}\right)=\sum_{k_{1}, \ldots, k_{d} \geq 0} c_{k_{1}, \ldots, k_{d}} \operatorname{wal}\left(k_{1}, x_{1}\right) \cdots \operatorname{wal}\left(k_{d}, x_{d}\right) .
$$


We have

$$
\begin{array}{rl}
\int_{[0,1]^{d}} & f\left(x_{1}, \ldots, x_{d}\right) d x_{1} \ldots d x_{d} \\
= & 2^{d-1}\left(\int_{[0,1 / 2]^{d}} f\left(x_{1}, \ldots, x_{d}\right) d x_{1} \ldots d x_{d}\right. \\
& \left.+\int_{[1 / 2,1]^{d}} f\left(x_{1}, \ldots, x_{d}\right) d x_{1} \ldots d x_{d}\right)-\Delta_{d}
\end{array}
$$

where

$$
\Delta_{d}=\sum_{\begin{array}{c}
0 \leq k_{1}, \ldots, k_{d} \leq 1 \\
\left(k_{1}, \ldots, k_{d}\right) \neq(0, \ldots, 0) \\
k_{1}+\cdots+k_{d}=0 \quad(\bmod 2)
\end{array}} c_{k_{1}, \ldots, k_{d}}
$$

Proof. First, we have

$$
\int_{[0,1]^{d}} f\left(x_{1}, \ldots, x_{d}\right) d x_{1} \ldots d x_{d}=c_{0, \ldots, 0} .
$$

Since

$$
\int_{0}^{1 / 2} \operatorname{wal}(1, x) d x=-\int_{1 / 2}^{1} \operatorname{wal}(1, x) d x=\frac{1}{2}
$$

and

$$
\int_{0}^{1 / 2} \operatorname{wal}(k, x) d x=\int_{1 / 2}^{1} \operatorname{wal}(k, x) d x=0
$$

for any $k \geq 2$, we have

$$
\int_{[0,1 / 2]^{d}} f\left(x_{1}, \ldots, x_{d}\right) d x_{1} \ldots d x_{d}=\frac{1}{2^{d}} \sum_{0 \leq k_{1}, \ldots, k_{d} \leq 1} c_{k_{1}, \ldots, k_{d}},
$$

and

$$
\int_{[1 / 2,1]^{d}} f\left(x_{1}, \ldots, x_{d}\right) d x_{1} \ldots d x_{d}=\frac{1}{2^{d}} \sum_{0 \leq k_{1}, \ldots, k_{d} \leq 1}(-1)^{k_{1}+\cdots+k_{d}} c_{k_{1}, \ldots, k_{d}} .
$$

Thus, the proof is complete.

We now consider a class of functions for which $\Delta_{d}=0$. First, we give some definitions. 
Definition 2. Let $m \geq 1$ be an integer and let $u$ be a non-empty subset $\left\{j_{1}, \ldots, j_{|u|}\right\} \subseteq$ $\{1, \ldots, d\}$. We define

$$
\phi_{u, m}\left(x_{1}, \ldots, x_{d}\right)=\prod_{i=1}^{|u|} \operatorname{wal}\left(k_{j_{i}}^{(m)}, x_{j_{i}}\right),
$$

for $2^{m-1} \leq k_{j_{1}}^{(m)}, \ldots, k_{j_{|u|}}^{(m)}<2^{m}$.

Definition 3. We define a class $\mathfrak{F}_{d}$ which consists of functions

$$
f\left(x_{1}, \ldots, x_{d}\right)=c_{0}+\sum_{\substack{1 \leq|u| \leq d \\|u| \text { is odd }}} \sum_{m=1}^{\infty} c_{u, m} \phi_{u, m}\left(x_{1}, \ldots, x_{d}\right),
$$

where $c_{0}$ and $c_{u, m}, m=1,2, \ldots$, are constants satisfying

$$
\left|c_{0}\right|+\sum_{\substack{1 \leq|u| \leq d \\|u| \text { is odd }}} \sum_{m=1}^{\infty}\left|c_{u, m}\right| 2^{m-1} \leq M<\infty .
$$

Here, $M$ is a constant.

We have the following theorem.

THEOREM 3. For any function $f\left(x_{1}, \ldots, x_{d}\right)$ in $\mathfrak{F}_{d}$, we have

$$
\begin{aligned}
\int_{[0,1]^{d}} f\left(x_{1}, \ldots, x_{d}\right) d x_{1} \ldots d x_{d}= & 2^{d-1}\left(\int_{[0,1 / 2]^{d}} f\left(x_{1}, \ldots, x_{d}\right) d x_{1} \ldots d x_{d}\right. \\
& \left.+\int_{[1 / 2,1]^{d}} f\left(x_{1}, \ldots, x_{d}\right) d x_{1} \ldots d x_{d}\right) .
\end{aligned}
$$

Proof. From equation (4) and Definition 3, it follows that the quantity $\Delta_{d}$ in Theorem 2 becomes zero for the case of $\mathfrak{F}_{d}$. Thus, the proof is complete.

\subsection{Convergence rate for the high-dimensional integration}

Hereafter, we denote by $\mathfrak{S}_{d}$ a class of $(t, d)$-sequences in base two whose generator matrices are non-singular and lower-triangular. Note that this class is a special subset of the class of non-uniform $(\infty, d)$-sequences introduced in Proposition 2. We prove the following theorem. 
THEOREM 4. For any sequence $\mathbf{s}_{n}, n=0,1, \ldots$, in the class $\mathfrak{S}_{d}$, the integration error $e_{N}$ of any function $f$ in $\mathfrak{F}_{d}$ is given by

$$
e_{N}\left(\mathbf{s}_{n}, f\right):=\left|I(f)-\frac{1}{N} \sum_{n=0}^{N-1} f\left(\mathbf{s}_{n}\right)\right|<\frac{1}{N} \sum_{\substack{1 \leq|u| \leq d \\|u| \text { is odd }}} \sum_{m=1}^{\infty}\left|c_{u, m}\right| \min \left(2^{m-1}, N\right) .
$$

From the condition in Definition 3, we have $e_{N}\left(\mathbf{s}_{n}, f\right)=O\left(N^{-1}\right)$, where the asymptotic constant is $M$. In order to prove the above theorem, we need the following three lemmas, which are generalizations of Lemmas 2, 3 and 4 in [8]. Let $s_{n}, n=0,1, \ldots$, be a sequence in $\mathfrak{S}_{1}$, and let $s_{n, m}$ denote the $m$ th bit of $s_{n}$.

Lemma 1. Suppose that $m \geq 1$. For all $n=h 2^{m}, h=0,1, \ldots$, and for all $0 \leq \ell<2^{m-1}$, we have

$$
s_{n+\ell, m}=1-s_{n+\ell+2^{m-1}, m} .
$$

Proof. If we compare $n+\ell$ and $n+\ell+2^{m-1}$, only the coefficient of $2^{m-1}$ in their binary representation is different while all of the others are identical. Since the generator matrix is non-singular and lower-triangular, this completes the proof.

Lemma 2. Suppose that $m \geq 1$. For all $n=h 2^{m}, h=0,1, \ldots$, and for all $0 \leq \ell<2^{m-1}$, we have

$$
\operatorname{wal}\left(k, s_{n+\ell}\right)=-\operatorname{wal}\left(k, s_{n+\ell+2^{m-1}}\right),
$$

where $k$ is any integer with $2^{m-1} \leq k<2^{m}$.

Proof. Since the generator matrix is non-singular and lower-triangular, we have $s_{n+\ell, e}=s_{n+\ell+2^{m-1}, e}$ for all $1 \leq e<m$. From the assumption that $k=b_{0}+$ $b_{1} 2+\cdots+b_{m-1} 2^{m-1}$ with $b_{m-1}=1$ and Lemma 1 , the proof is complete.

For $d$ dimensions, we denote a sequence in $\mathfrak{S}_{d}$ by $\mathbf{s}_{n}=\left(s_{n}^{(1)}, \ldots, s_{n}^{(d)}\right), n=$ $0,1, \ldots$, Then we have the following lemmas.

Lemma 3. Suppose that $m \geq 1$. Let $u$ be any non-empty subset $\left\{j_{1}, \ldots, j_{|u|}\right\} \subseteq$ $\{1, \ldots, d\}$. If the cardinality $|u|$ is odd, then for all $n=h 2^{m}, h=0,1, \ldots$, and for all $0 \leq \ell<2^{m-1}$, we have

$$
\phi_{u, m}\left(\mathbf{s}_{n+\ell}\right)=-\phi_{u, m}\left(\mathbf{s}_{n+\ell+2^{m-1}}\right)
$$

Proof. Denote

$$
\phi_{u, m}\left(\mathbf{s}_{n+\ell}\right)=\prod_{i=1}^{|u|} \operatorname{wal}\left(k_{j_{i}}^{(m)}, s_{n+\ell}^{\left(j_{j}\right)}\right)
$$


where $2^{m-1} \leq k_{j_{1}}^{(m)}, \ldots, k_{j_{|u|}}^{(m)}<2^{m}$. Without loss of generality, we assume that the number of $j_{i}, 1 \leq i \leq|u|$, with $\operatorname{wal}\left(k_{j_{i}}^{(m)}, s_{n+\ell}^{\left(j_{i}\right)}\right)=-1$ is odd. Then, from Lemma 2, the number of $j_{i}, 1 \leq i \leq|u|$, with $\operatorname{wal}\left(k_{j_{i}}^{(m)}, s_{n+\ell+2^{m-1}}^{\left(j_{i}\right)}\right)=-1$ is even because $|u|$ is odd. Thus, the proof is complete.

Note that $I\left(\phi_{u, m}\right)=0$ for any $u \neq \varnothing$ and $m \geq 1$. From Lemma 3, it follows that the integration error $e_{N}$ of $\phi_{u, m}$ with $|u|$ odd for any sequence $\mathbf{s}_{n}, n=0,1, \ldots$, in $\mathfrak{S}_{d}$ is given by

$$
e_{N}\left(\mathbf{s}_{n}, \phi_{u, m}\right)=\left|\frac{1}{N} \sum_{n=0}^{N-1} \phi_{u, m}\left(\mathbf{s}_{n}\right)\right|=\left|\frac{1}{N} \sum_{n=N-N_{m}}^{N-1} \phi_{u, m}\left(\mathbf{s}_{n}\right)\right| \leq \frac{\min \left(2^{m-1}, N\right)}{N}
$$

where $N_{m}$ is the residue of $N$ modulo $2^{m}$.

We are now ready to prove Theorem 4:

$$
\begin{aligned}
e_{N}\left(\mathbf{s}_{n}, f\right) & =\left|\frac{1}{N} \sum_{n=0}^{N-1} f\left(\mathbf{s}_{n}\right)-I(f)\right|=\left|\frac{1}{N} \sum_{n=0}^{N-1}\left(f\left(\mathbf{s}_{n}\right)-c_{0}\right)\right| \\
& =\left|\frac{1}{N} \sum_{n=0}^{N-1} \sum_{\substack{1 \leq|u| \leq d \\
|u| \text { is odd }}} \sum_{m=1}^{\infty} c_{u, m} \phi_{u, m}\left(\mathbf{s}_{n}\right)\right| \leq \sum_{\substack{1 \leq|u| \leq d \\
|u| \text { is odd }}} \sum_{m=1}^{\infty}\left|\frac{c_{u, m}}{N} \sum_{n=0}^{N-1} \phi_{u, m}\left(\mathbf{s}_{n}\right)\right| \\
& \leq \frac{1}{N} \sum_{\substack{1 \leq|u| \leq d \\
|u| \text { is odd }}} \sum_{m=1}^{\infty}\left|c_{u, m}\right| \min \left(2^{m-1}, N\right) .
\end{aligned}
$$

Thus, the proof is complete.

We should note that a sequence $\mathbf{s}_{n}, n=1,2, \ldots$, in $\mathfrak{S}_{d}$ is distributed in the sub-domain $\left[1 / 2^{m}, 1 / 2^{m-1}\right]^{d}$ when $n$ is a multiple of $2^{m-1}$ but not of $2^{m}$ for some $m \geq 1$. Therefore, high-discrepancy sequences employed in the above are essentially non-uniform because they are distributed in only a part of the diagonal domain $[0,1 / 2]^{d} \cup[1 / 2,1]^{d}$. 


\section{Discussion}

For a function $f\left(x_{1}, \ldots, x_{d}\right)$ which satisfies

$$
\begin{array}{rl}
\int_{[0,1]^{d}} & f\left(x_{1}, \ldots, x_{d}\right) d x_{1} \ldots d x_{d} \\
= & 2^{d-1}\left(\int_{[0,1 / 2]^{d}} f\left(x_{1}, \ldots, x_{d}\right) d x_{1} \ldots d x_{d}\right. \\
& \left.+\int_{[1 / 2,1]^{d}} f\left(x_{1}, \ldots, x_{d}\right) d x_{1} \ldots d x_{d}\right)
\end{array}
$$

there are three approaches to its numerical integration when the dimension $d$ is large.

- Monte Carlo methods. We have two types of Monte Carlo methods: uniform random sampling over the whole domain $[0,1]^{d}$ or over two sub-domains, $\left[0, \frac{1}{2}\right]^{d}$ and $\left[\frac{1}{2}, 1\right]^{d}$. For both cases, the convergence rate is $O\left(N^{-1 / 2}\right)$.

- Quasi-Monte Carlo or low-discrepancy methods. This approach provides the convergence rate $O\left((\log N)^{d} / N\right)$ for functions of bounded variation $[\mathbf{1}, \mathbf{4 - 7}$, 10].

- High-discrepancy methods. There are two types of high-discrepancy methods: The first type is to apply low-discrepancy sequences to each of the two subdomains, $\left[0, \frac{1}{2}\right]^{d}$ and $\left[\frac{1}{2}, 1\right]^{d}$, as the whole domain. The class of $(\infty, d)$ sequences introduced in Proposition 2 can be used for such a purpose. In this case, we get the convergence rate $O\left((\log N)^{d} / N\right)$ for functions of bounded variation. Note that this type is closely related to Hlawka-Mück approach [2]. The second type uses essentially non-uniform sequences such as the class $\mathfrak{S}_{d}$. For the particular case of $\mathfrak{F}_{d}$, we have shown the $O\left(N^{-1}\right)$ convergence rate, which is better than Monte Carlo and quasi-Monte Carlo methods. However, at least at present, nothing is known for functions besides $\mathfrak{F}_{d}$.

An important consequence from our result is that the 'low-discrepancy' approach is not the only way for accelerating the computation of high-dimensional numerical integration. If one considers a class of functions for which the uniform distribution is not a necessary condition to satisfy equation (1), there is a possibility to develop faster algorithms based on the 'high-discrepancy' approach than Monte Carlo and/or quasi-Monte Carlo (low-discrepancy) methods. There are several applications in practice involving a class of functions in high dimensions which are localized in a 
very limited domain. Commonly, we have used the so-called importance sampling techniques including Markov chain Monte Carlo methods for these applications. Unfortunately, all Monte Carlo techniques suffer from the slow convergence rate $O\left(N^{-1 / 2}\right)$, while our high-discrepancy approach, which is deterministic, has a potential of the convergence rate $O\left(N^{-1}\right)$. Therefore, we can consider the highdiscrepancy approach as a possible alternative. However, there are many topics to be explored in this new area.

\title{
REFERENCES
}

[1] M. Drmota and R. F. Tichy. Sequences, Discrepancies and Applications (Lecture Notes in Mathematics, 1651). Springer, Berlin, 1997.

[2] E. Hlawka and R. Mück. A transformation of equidistributed sequences. Applications of Number Theory to Numerical Analysis, S. K. Zaremba (ed.). Academic Press, New York, 1972, pp. 371-388.

[3] L. Kuipers and H. Niederreiter. Uniform Distribution of Sequences. Wiley, New York, 1974.

[4] J. Matoušek. Geometric Discrepancy: An Illustrated Guide. Springer, Berlin, 1999.

[5] H. Niederreiter. Random Number Generation and Quasi-Monte Carlo Methods (CBMS-NSF Regional Conference Series in Applied Mathematics, 63). SIAM, Philadelphia, PA, 1992.

[6] I. H. Sloan and S. Joe. Lattice Methods for Multiple Integration. Claredon Press, Oxford, 1994.

[7] S. Tezuka. Uniform Random Numbers: Theory and Practice. Kluwer Academic, Dordrecht, 1995.

[8] S. Tezuka. On the necessity of low-effective dimension. J. Complexity 21 (2005), 710-721.

[9] S. Tezuka and A. Papageorgiou. Exact cubature for a class of functions of maximum effective dimension. J. Complexity 22 (2006), 652-659.

[10] J. F. Traub and A. G. Werschulz. Complexity and Information. Cambridge University Press, Cambridge, 1998

\author{
Shu Tezuka \\ Faculty of Mathematics \\ Kyushu University \\ 6-10-1 Hakozaki \\ Higashi-ku \\ Fukuoka-shi \\ Fukuoka-ken 812-8581 \\ Japan \\ (E-mail:tezuka@math.kyushu-u.ac.jp)
}

